\title{
Maturitas Review
}

Managing menopausal symptoms after breast cancer - a multidisciplinary approach

Authors

Paul A. Cohen

Annabelle Brennan

Jennifer L. Marino

Christobel M. Saunders

Martha Hickey

\section{Conflict of Interest Statement:}

All authors have no conflict of interest to declare. 


\section{TITLE PAGE}

\section{Managing menopausal symptoms after breast cancer - a multidisciplinary approach}

\section{Authors}

Paul A. COHEN ${ }^{a, b, c}$, Annabelle BRENNAN ${ }^{e}$, Jennifer L. MARINO ${ }^{d, e}$, Christobel M. SAUNDERS ${ }^{f}$, Martha HICKEYd,e

a St. John of God Hospital Bendat Family Comprehensive Cancer Centre, 12 Salvado Road, Subiaco, Western Australia 6008, Australia.

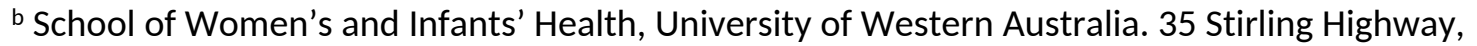
Crawley, Western Australia 6009, Australia.

c Institute for Health Research, University of Notre Dame Australia. 32 Mouat Street Fremantle, Western Australia 6160, Australia.

d Department of Obstetrics and Gynecology, The University of Melbourne, Parkville, Victoria 3052, Australia.

e Department of Obstetrics and Gynecology, Royal Women's Hospital, 20 Flemington Road, Parkville, Victoria 3052, Australia.

${ }^{\mathrm{f}}$ School of Surgery, The University of Western Australia, Crawley, Western Australia, Australia.

\section{Corresponding author's details:}

Dr Paul Cohen

St. John of God Hospital

Bendat Family Comprehensive Cancer Centre

12 Salvado Road

Subiaco, Western Australia, Australia

(M) +61 406888339

(E) Paul.Cohen@sjog.org.au 


\section{Managing menopausal symptoms after breast cancer - a multidisciplinary approach}

\section{ABSTRACT}

More than 6 million women worldwide are living with a past diagnosis of breast cancer. Most survive their illness and management of long-term consequences of treatment has become a priority in cancer care. Menopausal symptoms affect most breast cancer survivors and may significantly impair quality of life. We describe a multidisciplinary model to evaluate and manage these women using a patient-focused approach. The 'Multidisciplinary Menopause After Cancer Clinic' includes gynecologists, endocrinologists, GPs, a psychologist and a clinical nurse specialist. Benefits of this model include improved coordination of patient care, education, communication and evidencebased decision making.

\section{What we know:}

1. Menopausal symptoms after breast cancer may be more severe compared to women experiencing natural menopause.

2. Menopausal symptoms may lead to decreased compliance with adjuvant hormonal therapy.

3. Non-hormonal treatments are available for managing vasomotor symptoms after breast cancer

4. A multidisciplinary approach is associated with improved outcomes for women with breast cancer.

\section{What we think we know:}

1. Systemic menopausal hormone therapy (MHT) should be avoided after breast cancer

2. Topical vaginal estrogen may be safe in women with estrogen sensitive tumors on Tamoxifen and in women with estrogen-negative tumors.

\section{What we don't know:}

1. Whether low level systemic absorption of vaginal estrogen is clinically significant after breast cancer.

2. The optimum non-hormonal approach to managing menopausal symptoms after breast cancer

3. Whether testosterone is safe after breast cancer 
Managing menopausal symptoms after breast cancer - a multidisciplinary approach

\section{Background}

Breast cancer is the most common cancer in women worldwide, with 1.67 million new cases estimated in 2012 and a $4.6 \%$ lifetime risk (1). Most breast cancers are diagnosed in postmenopausal women with approximately two thirds experiencing hot flushes, half of whom report that their flushes are severe (2). Since the findings of a randomized trial which showed that Menopausal Hormone Therapy (MHT) was associated with more than a threefold increase in recurrent disease (3), women diagnosed with breast cancer who are using MHT will be advised to immediately discontinue treatment. Cessation of MHT commonly leads to a resurgence of hot flushes. Furthermore, most breast cancers express the estrogen-receptor and will be treated with anti-estrogen therapies which commonly exacerbate menopausal symptoms and these symptoms may lead to decreased compliance with cancer therapy, adversely affecting prognosis (4). Premenopausal women with breast cancer are often treated with chemotherapy which may induce premature or early menopause (5). In those managed with bilateral oophorectomy, $>90 \%$ have hot flushes and vaginal dryness which may be more severe and prolonged than those experienced by women following natural menopause. Early menopause can have an adverse impact on quality of life, sexual function and self-esteem (6). Menopausal symptoms represent the most common and troublesome side-effects of breast cancer treatment for women of all ages - an observation which is of increasing clinical significance as survival from breast cancer continues to improve.

The objective of this review is to describe a unique multidisciplinary approach to managing menopausal symptoms, health and wellbeing after breast cancer. Discussion of the nonpharmacologic and pharmacologic treatment of vasomotor symptoms is beyond the scope of the review and the reader is referred to the 2016 Cancer Australia Guidelines 'Management of menopausal women with a history of breast cancer' (7).

\subsection{Breast cancer treatment and bone health}

Breast cancer treatment may adversely affect bone health. In a meta-analysis of 31,920 postmenopausal women with estrogen-receptor positive early breast cancer randomized to aromatase inhibitors (Al) or tamoxifen, there was a $42 \%$ relative risk increase of bone fractures in women allocated to an Al during the first 4 years of treatment (8). This risk remained significantly elevated 
during subsequent follow up (years 5 - 9). Reduced breast cancer recurrence with Als compared to tamoxifen (8) has made them first line endocrine therapy after ER +ve postmenopausal breast cancer, and this will have major implications for bone health in survivors.

\subsection{Breast cancer and cardiovascular health}

As greater numbers of women survive breast cancer understanding how treatment may affect longterm health becomes more important. Evidence is emerging, but several breast cancer therapies including chest wall radiation, systemic chemotherapy, HER2-antagonists and aromatase inhibitors are potentially cardiotoxic (9). Furthermore, cardiovascular disease (CVD) and breast cancer share common risk factors such as obesity and the metabolic syndrome, so women diagnosed with breast cancer may be at increased baseline risk of CVD (10). Similarly, BRCA gene mutation carriers may be at higher risk of CVD due to the intrinsic role of the BRCA proteins in normal cardiac function and structure (11). Chemotherapy and radiation treatment of breast cancer may also increase CVD (9). Cardiac surveillance using 3D ultrasound echocardiography and MRI, has been proposed in second round chemotherapy and/or prior to commencement of trastuzamab to identify those at elevated risk of CVD in order to individualize treatment and minimize cardiovascular morbidity (9).

\subsection{Sexuality and breast cancer}

A breast cancer diagnosis and its treatment may adversely affect sexual function. Breast surgery may have a significant effect on a woman's sexual experience. In a cross-sectional study of 269 breast cancer patients who underwent breast surgery at an academic oncology center, patients who were more satisfied with their chest appearance had significantly better sexual function than those who were not (12). Surgical approach appeared to correlate with sexual experience, with participants undergoing lumpectomy almost twice as likely to experience breast-specific pleasure compared to mastectomy and reconstruction. The risk of 'severe pain' during sexual intercourse was twice as common in women after chemotherapy for breast cancer compared to those who had not received chemotherapy (10). Als are associated with urogenital atrophy which may negatively impact on quality of life and sexual function.

\subsection{Breast cancer and psychological sequelae}

Mood disorders including depression and anxiety are increased over the menopause transition and may be further increased following a diagnosis of breast cancer. A recent systematic review of 17 studies which included 10,522 patients and 1977 controls, suggested a higher prevalence of depressive symptoms among breast cancer survivors compared to the general population (13). 


\subsection{Breast cancer and cognition}

Whether breast cancer treatment leads to long-term cognitive impairment remains unclear and studies have reported mixed findings (14-16). Recent imaging studies suggest that chemotherapy for breast cancer may affect white matter integrity, but the clinical significance of this is uncertain (17). Studies are required to determine which breast cancer treatments may affect cognitive function, how to identify patients at risk of cognitive impairment and to individualize treatment to minimize this risk. There is a need for effective interventions to preserve cognitive function and to treat cognitive decline in breast cancer patients (18).

\subsection{Sleep disturbance}

Sleep disturbance is common in postmenopausal women and is highly prevalent in breast cancer survivors (19). Its etiology is poorly understood but may include demographic, environmental and lifestyle factors, psychological disturbances, vasomotor symptoms and comorbid medical disorders as well as treatment-related side effects (20). Managing sleep disturbance is a priority in cancer care since sleep difficulties impair quality of life, increase co-morbidities such as fatigue, anxiety, depressive symptoms and poor social functioning (21).

\subsection{Supporting survivors in the workplace}

Effective treatment of breast cancer and more women in the workforce mean that a higher proportion of survivors can expect to return to paid employment after breast cancer (22). A recent systematic review identified barriers and facilitators affecting return to work (23). Barriers include treatment with chemotherapy; heavy physical work; poor general health and fatigue as well as psychological factors such as depression and distress. Facilitators include social, family and employer support and financial independence. Several targeted interventions to enable return to work after breast cancer have been developed, but more information is needed about which interventions are most effective (24).

\section{The multidisciplinary Menopause Symptoms After Cancer Clinic}

A novel multidisciplinary model of care to manage menopausal symptoms in cancer patients was developed in Australia in 2003 and has been replicated elsewhere in Australia and internationally (25). The service is set within a large public women's hospital and is staffed by gynecologists, an endocrinologist, family physicians, a clinical psychologist and a clinical nurse specialist. Service design and delivery is based on the principal that a multidisciplinary approach to cancer care with 
individualized treatment plans decreases mortality and enhances quality of life $(26,27)$. Without this approach, management of menopausal symptoms may not be coordinated between primary and tertiary care providers and women may receive conflicting information regarding safe and effective treatments. Women with breast cancer attending the MSAC receive evidence-based information regarding non-pharmacological and non-hormonal treatments and, where appropriate, topical vaginal treatments (25). Consensus protocols and regular meetings with breast surgeons and oncologists mean that women are given consistent treatment advice and support. Those women with clinical risk factors for osteoporosis are also offered dual energy $\mathrm{x}$-ray absorptiometry assessment of bone mineral density. Where indicated, patients are also referred to urogynecology, clinical genetics, clinical psychology, psychiatry, or fertility services. Post-menopausal women taking tamoxifen with vaginal bleeding are investigated by pelvic ultrasonography, endometrial biopsy and/or curettage to exclude endometrial hyperplasia or malignancy.

\subsection{Role of the Clinical Nurse Specialist (CNS)}

Development of the CNS role is a key focus of this multidisciplinary model of care. The role is undertaken by a senior registered nurse jointly trained in breast cancer and menopause. The CNS reviews all patients and provides information about menopause and midlife health, behavioral and lifestyle strategies for reducing symptoms, and information about optimizing psychological and sexual function. General midlife health issues such as diet, weight control, and exercise, are discussed which may improve long-term health and quality of life in breast cancer patients. The CNS also provides information sheets to patients and links to national organizations which provide information and support.

\section{Unanswered Questions}

\subsection{Systemic estrogen}

A randomized controlled trial to assess the safety of MHT after breast cancer (HABITS: hormonal treatment after breast cancer - is it safe?) (3) was terminated early due to an elevated recurrence in MHT users (HR 3.5; 95\% Cl 1.5 - 8.1). A parallel study in Stockholm was also terminated early, although this association was not seen (28). Potentially, the difference in findings between these studies could be due to greater use of combined MHT in the HABITS study and estrogen alone in the Stockholm trial, but further randomised studies of MHT after breast cancer are unlikely to be conducted. 
Similarly, a randomized controlled trial of tibolone vs placebo after breast cancer (LIBERATE) demonstrated an increased recurrence in tibolone users (HR 1.44; $95 \% \mathrm{Cl} 1.16$ - 1.79) so tibolone should also be avoided after breast cancer (29).

In clinical practice MHT is avoided after breast cancer. Rarely, MHT may be considered in cases where symptoms are severe, significantly impair quality of life and are refractory to non-hormonal treatments. Patients should be advised that MHT may increase the risk of breast cancer recurrence and the treating oncology team should be involved in the decision. Due to the heterogeneity of the disease it is important to consider stage, tumor histology, lymph node status and risk of recurrence when deciding on the use of MHT after a breast cancer diagnosis. Prior to commencing MHT it is important to assess the woman's cardiovascular risk and to consider the relative and absolute contra-indications such as a history of ischemic heart disease.

\subsection{Topical vaginal estrogen}

There are no high-quality studies demonstrating the safety of vaginal estrogen in women with a history of breast cancer. Vaginal estradiol and estriol are systemically absorbed at low levels but the clinical significance of this is unknown. Vaginal dryness leading to pain during sexual activity is common after breast cancer. In a randomized trial comparing silicone with water based lubricants, the silicone product reduced pain with intercourse more effectively than the water based lubricant (30).

Vaginal estrogens may be considered for persistent vulvo-vaginal symptoms in breast cancer survivors when non-hormonal treatments are ineffective.

\subsection{Testosterone}

Testosterone supplementation has not been shown to be effective in female cancer survivors with reduced libido (31). High quality data to support the safety of testosterone replacement in breast cancer survivors are lacking. A randomized phase III trial investigating the efficacy of testosterone compared to placebo in treating postmenopausal breast cancer patients with arthralgia due to Anastrozole or Letrozole is currently recruiting (32). Decisions regarding the use of testosterone after breast cancer should be taken in conjunction with the woman's treating oncologist.

\subsection{Compounded 'bioidentical' hormones}

Compounded hormones have the same chemical structure as endogenous hormones. They may contain high concentrations of sex steroids and carry similar risks to pharmacological hormone 
products. There are no studies of compounded hormones in women with breast cancer and they should be avoided.

\section{Summary}

Menopausal symptoms are common after breast cancer and may be more problematic compared to women experiencing natural menopause (33). These symptoms can impair quality of life and result in decreased compliance with adjuvant hormonal therapies which may adversely affect prognosis. A multidisciplinary model of care is associated with improved outcomes for cancer survivors (34).

\section{Practice Points}

- Menopausal symptoms are common after breast cancer treatment

- A multidisciplinary model of care can optimize symptom management and long-term health

\section{Research Agenda}

High quality studies are required to investigate

- Whether vaginal estrogens and selective estrogen receptor modulators are safe and effective in breast cancer patients

\section{Contributors}

All authors contributed to the preparation and editing of the manuscript.

\section{Conflict of interest}

The authors declare that they have no conflict of interest.

\section{Funding}

No funding source to declare.

\section{Provenance and peer review}

\section{References}


1. Ferlay J, Soerjomataram I, Dikshit R, Eser S, Mathers C, Rebelo M, et al. Cancer incidence and mortality worldwide: sources, methods and major patterns in GLOBOCAN 2012. International journal of cancer. 2015;136(5):E359-86.

2. Carpenter JS, Rand KL. Modeling the hot flash experience in breast cancer survivors. Menopause. 2008;15(3):469-75.

3. Holmberg L, Iversen $\mathrm{OE}$, Rudenstam CM, Hammar M, Kumpulainen E, Jaskiewicz J, et al. Increased risk of recurrence after hormone replacement therapy in breast cancer survivors. Journal of the National Cancer Institute. 2008;100(7):475-82.

4. Meggetto O, Maunsell E, Chlebowski R, Goss P, Tu D, Richardson H. Factors Associated With Early Discontinuation of Study Treatment in the Mammary Prevention.3 Breast Cancer Chemoprevention Trial. Journal of clinical oncology : official journal of the American Society of Clinical Oncology. 2017;35(6):629-35.

5. Goodwin PJ, Ennis M, Pritchard KI, Trudeau M, Hood N. Risk of menopause during the first year after breast cancer diagnosis. Journal of clinical oncology : official journal of the American Society of Clinical Oncology. 1999;17(8):2365-70.

6. Schover LR. Premature ovarian failure and its consequences: vasomotor symptoms, sexuality, and fertility. Journal of clinical oncology : official journal of the American Society of Clinical Oncology. 2008;26(5):753-8.

7. Cancer Australia. Management of menopausal symptoms in women with a

history of breast cancer. URL: https://canceraustralia.gov.au/clinical-best-practice/breastcancer/management-menopausal-symptoms-women-history-breast-cancer-\%E2\%80\%93-clinicalpractice-guideline Accessed March 3 2017. 2016.

8. (EBCTCG) EBCTCG. Aromatase inhibitors versus tamoxifen in early breast cancer: patientlevel meta-analysis of the randomised trials. The Lancet. 2015;386(10001):1341-52.

9. Maas AH, Ottevanger N, Atsma F, Cramer MJ, Leiner T, Poortmans P. Cardiovascular surveillance in breast cancer treatment: A more individualized approach is needed. Maturitas. 2016;89:58-62.

10. Abdel-Qadir H, Austin PC, Lee DS, Amir E, Tu JV, Thavendiranathan P, et al. A PopulationBased Study of Cardiovascular Mortality Following Early-Stage Breast Cancer. JAMA cardiology. 2017;2(1):88-93.

11. van Westerop LL, Arts-de Jong M, Hoogerbrugge N, de Hullu JA, Maas AH. Cardiovascular risk of BRCA1/2 mutation carriers: A review. Maturitas. 2016;91:135-9.

12. S. Pesek MO, S. Fogarty, A. Stuckey, C. Raker, M. Clark, E., Kunkel JG. Sexual Function After Breast Cancer Surgery. Abstracts/Gynecol Oncol 2015;139:588.

13. Maass SW, Roorda C, Berendsen AJ, Verhaak PF, de Bock GH. The prevalence of long-term symptoms of depression and anxiety after breast cancer treatment: A systematic review. Maturitas. 2015;82(1):100-8.

14. Ramalho M, Fontes F, Ruano L, Pereira S, Lunet N. Cognitive impairment in the first year after breast cancer diagnosis: A prospective cohort study. Breast (Edinburgh, Scotland). 2017;32:173-8.

15. Jim HS, Phillips KM, Chait S, Faul LA, Popa MA, Lee YH, et al. Meta-analysis of cognitive functioning in breast cancer survivors previously treated with standard-dose chemotherapy. Journal of clinical oncology : official journal of the American Society of Clinical Oncology. 2012;30(29):357887.

16. Amidi A, Christensen S, Mehlsen M, Jensen AB, Pedersen AD, Zachariae R. Long-term subjective cognitive functioning following adjuvant systemic treatment: 7-9 years follow-up of a nationwide cohort of women treated for primary breast cancer. British journal of cancer.

2015;113(5):794-801. 
17. Menning S, de Ruiter MB, Veltman DJ, Boogerd W, Oldenburg HS, Reneman L, et al. Changes in brain white matter integrity after systemic treatment for breast cancer: a prospective longitudinal study. Brain Imaging Behav. 2017.

18. Treanor CJ, McMenamin UC, O'Neill RF, Cardwell CR, Clarke MJ, Cantwell M, et al. Nonpharmacological interventions for cognitive impairment due to systemic cancer treatment. The Cochrane database of systematic reviews. 2016(8):Cd011325.

19. Savard J, Morin CM. Insomnia in the context of cancer: a review of a neglected problem. Journal of clinical oncology : official journal of the American Society of Clinical Oncology. 2001;19(3):895-908.

20. Fiorentino L, Ancoli-Israel S. Insomnia and its treatment in women with breast cancer. Sleep medicine reviews. 2006;10(6):419-29.

21. Accortt EE, Bower JE, Stanton AL, Ganz PA. Depression and vasomotor symptoms in young breast cancer survivors: the mediating role of sleep disturbance. Archives of women's mental health. 2015;18(3):565-8.

22. Caron M, Durand MJ, Tremblay D. Perceptions of Breast Cancer Survivors on the Supporting Practices of Their Supervisors in the Return-to-Work Process: A Qualitative Descriptive Study. J Occup Rehabil. 2017.

23. Islam T, Dahlui M, Majid HA, Nahar AM, Mohd Taib NA, Su TT, et al. Factors associated with return to work of breast cancer survivors: a systematic review. BMC Public Health. 2014;14 Suppl 3:S8.

24. Bilodeau K, Tremblay D, Durand MJ. Exploration of return-to-work interventions for breast cancer patients: a scoping review. Supportive care in cancer : official journal of the Multinational Association of Supportive Care in Cancer. 2017.

25. Hickey M, Emery LI, Gregson J, Doherty DA, Saunders CM. The multidisciplinary management of menopausal symptoms after breast cancer: a unique model of care. Menopause. 2010;17(4):727-

33.

26. Fleissig A, Jenkins V, Catt S, Fallowfield L. Multidisciplinary teams in cancer care: are they effective in the UK? The Lancet Oncology. 2006;7(11):935-43.

27. Taylor C, Shewbridge A, Harris J, Green JS. Benefits of multidisciplinary teamwork in the management of breast cancer. Breast Cancer (Dove Med Press). 2013;5:79-85.

28. Fahlen $\mathrm{M}$, Fornander $\mathrm{T}$, Johansson $\mathrm{H}$, Johansson $\mathrm{U}$, Rutqvist LE, Wilking $\mathrm{N}$, et al. Hormone replacement therapy after breast cancer: 10 year follow up of the Stockholm randomised trial. European journal of cancer. 2013;49(1):52-9.

29. Kenemans P, Bundred NJ, Foidart JM, Kubista E, von Schoultz B, Sismondi P, et al. Safety and efficacy of tibolone in breast-cancer patients with vasomotor symptoms: a double-blind, randomised, non-inferiority trial. The Lancet Oncology. 2009;10(2):135-46.

30. Hickey M, Marino JL, Braat S, Wong S. A randomized, double-blind, crossover trial comparing a silicone- versus water-based lubricant for sexual discomfort after breast cancer. Breast cancer research and treatment. 2016;158(1):79-90.

31. Barton DL, Wender DB, Sloan JA, Dalton RJ, Balcueva EP, Atherton PJ, et al. Randomized controlled trial to evaluate transdermal testosterone in female cancer survivors with decreased libido; North Central Cancer Treatment Group protocol N02C3. Journal of the National Cancer Institute. 2007;99(9):672-9.

32. Testosterone in Treating Postmenopausal Patients With Arthralgia Caused by Adjuvant Aromatase Inhibitor Treatment. ClinicalTrials.gov Identifier: NCT01573442.

https://clinicaltrials.gov/ct2/show/NCT01573442 accessed April 62017.

33. Marino JL, Saunders CM, Emery LI, Green H, Doherty DA, Hickey M. Nature and severity of menopausal symptoms and their impact on quality of life and sexual function in cancer survivors compared with women without a cancer history. Menopause. 2014;21(3):267-74.

34. Studdert DM. Can liability rules keep pace with best practice? The case of multidisciplinary cancer care. Med J Aust. 2008;188(7):380-1. 
Managing menopausal symptoms after breast cancer - a multidisciplinary approach

\section{Highlights}

- Menopausal symptoms after breast cancer are common and may be severe

- Menopausal symptoms may lead to decreased compliance with adjuvant hormonal therapy

- Multidisciplinary care is associated with improved outcomes after breast cancer 